1 Bacon AS, Frazer DG, Dart JKG, Matheson M, Ficker LA, Wright $P$. A review of 72 consecutive cases of Acanthamoeba keratitis, 1984-1992. Eye 1993;7:719-25.

2 Larkin DF, Kilvington S, Dart JK. Treatment of Acanthamoeba keratitis with polyhexamethylene biguanide. Ophthalmology 1992;99:185-91.

3 Elder MJ, Dart JKG. Chemotherapy for Acanthamoeba keratitis. Lancet 1995;345:791-2.

4 Green K, Livingston V, Bowman K, Hull DS Chlorhexidine effects on corneal epithelium and endothelium. Arch Ophthalmol 1980;98:1273-8.

5 Berry M, Easty DL. Isolated human and rabbit eye: models of corneal toxicity. Toxicology in vitro 1993; $7: 461-4$.

6 Stickler DJ, Thomas B. Antiseptic and antibiotic resistance in Gram-negative bacteria causing urinary tract infection. $\mathcal{F}$ Clin Pathol 1980;33: 288-96.

7 Marrie TJ, Costerton JW. Prolonged survival of Serratia marcescens in chlorhexidine. Appl Environ Microbiol 1981;42:1093-102.

8 Bottone EJ, Madayag RM, Qureshi MN. Acanthamoeba keratitis: synergy between amebic and bacterial cocontaminants in contact lens care systems as a prelude to infection. $f$ Clin systems as a prelude to
Microbiol 1992;30:2447-50.

\section{Fuchs' heterochromic uveitis and sarcoidosis}

EdrToR,--Richard Goble and Philip Murray provoke further interest in the possible cause(s) of Fuchs' heterochromic uveitis (FHU), in reporting five patients with suggested sarcoidosis, including four with raised serum angiotensin converting enzyme (ACE). In patients with sarcoidosis, increased production of ACE is attributed to activated cells within granulomata. In 'granulomatous' uveitis it is reasonable, even in the absence of systemic symptoms, to investigate for the possibility of sarcoidosis. However, it is rarely appropriate to obtain biopsies of ocular tissue for this purpose. We therefore rely on indirect methods of diagnosis.

Weinreb ${ }^{2}$ measured ACE levels in normal controls and found that $4.2 \%$ had significantly raised levels. By comparison, of those with 'granulomatous' uveitis but without evidence of systemic sarcoidosis, $44 \%$ had raised levels. His conclusion, that 'ocular sarcoidosis' may be diagnosed in the absence of systemic evidence, is attractive, though unproved, and would explain a large subset of idiopathic uveitis. However, to extend this group to include forms of uveitis which are untypical of sarcoid related uveitis is mere speculation.

Iris nodules are a recognised feature of FHU, ${ }^{3}$ yet their appearance (small, domeshaped, multiple, and translucent) and position (on the anterior iris surface, mostly peripupillary, scattered symmetrically) differentiate them from nodules seen in granulomatous disease (usually larger, fewer, often irregular in shape, sometimes buried within the stroma). Their presence in FHU cannot per se make a convincing case for a granulomatous aetiology. Indeed, Rothova et $a l,{ }^{4}$ in reporting four black patients with FHU and iris nodules, investigated for sarcoidosis and found no evidence (including normal ACE levels in all four cases). There is no patient with systemic sarcoidosis in the 184 strong Manchester cohort of FHU patients (Jones NP, unpublished data). Also, mutton fat keratic precipitates as described in two of Goble and Murray's patients are not considered to be an accepted part of the FHU spectrum and their appearance should lead one to reconsider the diagnosis.

The evidence for the relevance of raised ACE levels in a small number of patients with possible FHU is therefore unconvincing. The test has significant problems with both specificity and sensitivity, and raised levels are reported in as many as $17 \%{ }^{5}$ in a population of non-sarcoid uveitis. The significance of raised ACE levels in four patients with possible FHU should therefore be statistically justified in the context of the authors' FHU group of patients. Even should this reach statistical significance, the assumption that this represents a form of sarcoidosis is speculative.

NP JONES

Deparment of Ophthalmology, Royal Eye Hospital, Oxford Road, Manchester M13 9WH

1 Goble RR, Murray PI. Fuchs' heterochromic uveitis and sarcoidosis. $\mathrm{Br} f$ Ophthalmol 1995;79:1021-3.

2 Weinreb RN, Kimura SJ. Uveitis associated with sarcoidosis and angiotensin converting enzyme. Am $₹$ Ophthalmol 1980;89:180-5.

3 Jones NP. Fuchs' heterochromic uveitis: a Jones NP. Fuchs heterochromic uveitis: a
reappraisal of the clinical spectrum. Eye 1991;5: reappraisal

4 Rothova A, La Hey E, Baarsma GS, Breebaart AC. Iris nodules in Fuchs' heterochromic uveitis. Am ₹ Ophthalmol 1994;118:338-42.

5 Neves RA, Rodrigues A, Power WJ, PedrosaSeres M, Foster CS. The value of combined serum angiotensin converting enzyme and gllium angion in the diconvering sis. In: Nus in the diagnosis of ocular sarcoidoRR, Geri I, eds. Advances in ocular immunology. Amsterdam: Elsevier, 1994:353-6.

\section{Reply}

EDTrOR,-We thank Nicholas Jones for his comments on our paper. $\mathrm{He}$ raises a number of important issues including the clinical characteristics of our patients and the difficulty in diagnosing ocular sarcoidosis when biopsy is not possible.

At some time point in their disease each of our five patients showed the characteristic clinical features of FHU. All patients had uniformly distributed, stellate keratic precipitates (KPs), iris stromal atrophy with heterochromia, and no posterior synechiae. Four out of five patients had iris nodules and three had posterior subcapsular cataract. Although mutton fat KPs are not a recognised feature of FHU, very occasionally these patients can develop a supra-added anterior uveitis resulting in the formation of atypical KPs.

Sarcoidosis can be difficult to diagnose particularly in the absence of extraocular features. Although elevated angiotensin converting enzyme (ACE) levels are not specific for sarcoidosis the uveitis in these patients would be highly unlikely to result from any of the other causes of a raised ACE. Also, one patient was Kveim positive and another had chest $x$ ray changes compatible with sarcoidosis.

We agree that a larger series of patients would be required in order to demonstrate any statistically significant association between raised ACE levels and FHU. Nevertheless, the finding of a raised ACE in four patients with a condition that forms only $3 \%$ of all uveitis entities would appear to be more than jus coincidental.

Ocular sarcoidosis may not always present with the typical textbook findings, an example of this would be those patients who have fundal appearance similar to birdshot retinochoroidopathy but are HLA-A29 negative. The features of FHU seen in our patients may be another atypical presentation.

Although FHU has been reported in combination with numerous conditions, a possible association with sarcoidosis has not been previously described. We felt that this was an interesting new finding which would support the theory that FHU may be a secondary phenomenon or a clinical end state of a number of conditions.

RR GOBLE

PI MURRAY

Academic Unit of Ophthalmology, Birmingham and Midland Eye Centre, City Hospital NHS Trust,

Dudley Road,

Birmingham B18 7QH

\section{Determining the importance of eye diseases in Africa}

EDITOR,-In Africa the public health importance of trachoma and xerophthalmia is often underestimated when based on routine surveillance data and even data from population based surveys of low vision and blindness. Surveillance data may under-represent occurrence because both diseases are prevalent in children who rarely complain of it and health personnel seldom examine patients' eyes if they do not have an eye complaint. Population based surveys may under-represent occurrence if cluster sampling is used as this is a weak technique for detecting diseases with focal distribution. These problems are highlighted below using experiences on estimating the importance of these diseases in Ethiopia.

In 1978-80 the Ethiopian Nutrition Institute and the WHO conducted a countrywide assessment on the reporting of xerophthalmia in health centres and hospitals. The study concluded that the condition was rarely recorded. ${ }^{1}$ In the early 1980 s two foci of vitamin A deficiencies were detected in faminefree areas of Arsi, Bale, and Gamu Gofa provinces. ${ }^{2}$ Trachoma was also heavily underreported. In a study of eye conditions at three health centres, where all children under 10 years of age attending the centres for any reason were examined for eye diseases, prevalence of trachoma was 10-fold higher than previously suggested by hospital records.

In 1981 a population based survey of blindness was undertaken. ${ }^{4}$ The survey found a blindness prevalence of between $1.35 \%$ and $1.5 \%$ and trachoma was found to be the major cause. However, the survey failed to detect a high prevalence of xerophthalmia in the country. By chance the xerophthalmia foci were not selected when the random sample of clusters were drawn.

Because of the limitation of the methods discussed above health authorities should identify areas where the population is likely to be at high risk, because of the presence of known risk factors for that condition, and then undertake a sample survey of the children within the high risk areas. This should provide a more realistic insight into the magnitude of the problem in specific high risk areas and direct planning for targeted intervention.

G DE SOLE

1 Pascoe Avenue, BP 6988, Harare, Zimbabwe

1 WHO, UNICEF, USAID, HKI, IVACG. Control of vitamin A deficiency and xerophthalmia. 1982.

2 Lindtiorn B. Xerophthalmia in Gardulla area of southwest Ethiopia. Ethiop Med ₹ 1983;21:16974.

3 De Sole G, Belay Y, Zegeye B. Vitamin A deficiency in southern Ethiopia. Am $\mathcal{f}$ Clin Nutr deficiency in south

4 Budden FH. Blindness in Ethiopia. WHO/STC unpublished report. Geneva: WHO, 1981. 